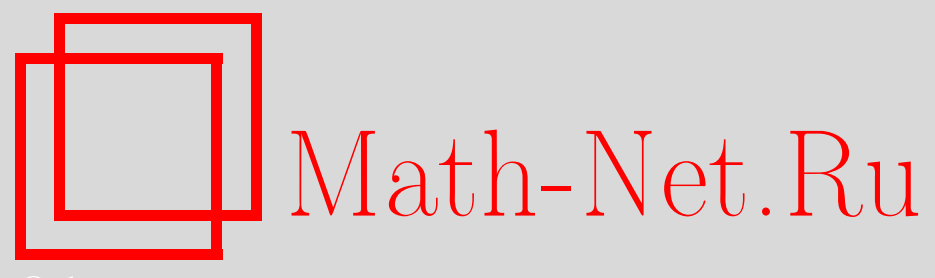

В. Г. Смирнов, Методы решения систем квазисимметрических уравнений и их применение к анализу генераторов с управляемым движением, Матем. вопр. криптогр., 2010, том 1, выпуск 3, 67-91

DOI: https://doi.org/10.4213/mvk16

Использование Общероссийского математического портала Math-Net.Ru подразумевает, что вы прочитали и согласны с пользовательским соглашением http://www . mathnet.ru/rus/agreement

Параметры загрузки:

IP : 3.95 .254 .165

26 апреля 2023 г., 12:34:05 


\title{
Методы решения систем квазисимметриче- ских уравнений и их применение к анализу генераторов с управляемым движением
}

\author{
Смирнов В. Г. \\ Академия криптографии Российской Федерации, Москва
}

Получено 22.IV.2010

Даются определения квазисимметрических функций и систем квазисимметрических уравнений. Применение метода, основанного на сведении к задаче о кратчайших путях, приводит к построению полиномиального алгоритма решения таких систем. Указана возможность решения систем с искажённой правой частью. Использование полученных результатов при анализе генераторов с управляемым движением расширяет область применимости алгебраических методов и позволяет повысить эффективность соответствующих атак.

Ключевые слова: системы квазисимметрических уравнений, алгоритмы поиска решений, генераторы с управляемым движением

\section{Methods of solution of systems of quasisymmetric equations with applications to the analysis of clock-controlled generators}

\section{G. Smirnov}

Academy of Cryptography of Russian Federation, Moscow

\begin{abstract}
Quasisymmetric functions and systems of quasisymmetric equations are defined. An algorithm of solution of such systems is based on the reduction to a shortest path problem. A possibility of solution of system with noise is discussed. Application of results to the analysis of clock-controlled generators expands the domain of algebraic methods and increase the effectiveness of corresponding attacks.
\end{abstract}

Key words: systems of quasisymmetric equations, algorithmic solutions, clock-controlled generators

Citation: Mathematical Aspects of Cryptography, 2010, vol. 1, no. 3, pp. 67-92 (Russian). 


\section{Введение}

Методы решения систем дискретных уравнений, использующие сведение исходной задачи к нахождению подграфа кратчайших путей некоторого орграфа, рассматривались в $[1,2,3]$. Они основаны на «погружении» левых частей рассматриваемой системы в модуль дискретных функций над кольцом целых чисел, порождаемый графом определённого вида. Если систему удаётся погрузить в модуль, порождаемый графом с числом вершин, полиномиальным относительно числа неизвестных, то существует полиномиальный алгоритм её решения.

Один из примеров существования полиномиального алгоритма связан с рассмотрением определяемых далее систем квазисимметрических уравнений. Их использование при анализе генераторов с управляемым движением расширяет границы применимости алгебраических методов и позволяет при некоторых условиях повысить эффективность алгебраических атак.

Остановимся на кратком содержании работы.

В п. 1 приводится сжатый и систематизированный обзор результатов предыдущих исследований, включающий некоторые новые результаты.

В п. 2 исследуется возможность применения $\Delta$-графов для решения систем дискретных уравнений, том числе - с искажённой правой частью. Даются необходимые определения и предлагается алгоритм построения графа решений системы, сложность которого полиномиально зависит от числа вершин $\Delta$-графа.

В п. 3 вводятся в рассмотрение два вида $\Delta$-графов с полиномиальным числом вершин, порождающих модули квазисимметрических функций, и исследуются некоторые их свойства. Рассматривается полиномиальный алгоритм решения систем квазисимметрических уравнений с искажённой правой частью.

В п. 4 анализируются две типичные схемы генераторов с управляемым движением, заданных над конечным простым полем. Показано, что функционирование таких генераторов описывается системами квазисимметрических уравнений. Предложен метод определения ключей генераторов по отрезку искажённой выходной последовательности, основанный на решении систем указанного вида.

\section{1. Исходные конструкции}

1.1. Обозначения. Введём некоторые обозначения, систематически используемые в дальнейшем изложении.

$$
\begin{aligned}
& A_{1} \times \ldots \times A_{n}-\text { прямое произведение множеств } A_{1}, \ldots, A_{n} ; \\
& A^{n}=\underbrace{A \times \ldots \times A}_{n \text { раз }} ;
\end{aligned}
$$


$a^{(n)}=\left(a_{1}, \ldots, a_{n}\right) ; a^{(n)} \in A^{n}$, если $a_{i} \in A, i=1, \ldots, n ;$

$\left[a_{1}, \ldots, a_{i}\right]_{k}=a_{1}+a_{2} k+\ldots+a_{i} k^{i-1}$;

$Z$ - кольцо целых чисел;

$Z_{k}=\{0,1, \ldots, k-1\}$, где $k \geq 2$;

$\mathrm{GF}(p)$ - конечное поле, содержащее $p$ элементов;

$c$ - функция, тождественно равная константе $c$;

символы $\underset{m}{+}, \frac{-}{m}$ означают операции сложения и вычитания по $\bmod m$; при $m=2$ для операции сложения используется символ $\oplus$.

1.2. Конечные дискретные функции. Далее рассматриваются конечные дискретные функции от $n$ переменных, задаваемые отображениями $f: M \rightarrow Z$, где $M \subseteq Z_{k}^{n}, k-$ некоторое фиксированное число, и множество $M$ содержит не менее двух элементов. Множество дискретных функций указанного вида при заданном множестве $M$ обозначим $D(M ; Z)$. Для функции от $n$ переменных при $n>1$ используется обозначение $f\left(x^{(n)}\right)$, где $x^{(n)} \in M$.

Множество значений, принимаемых функцией $f\left(x^{(n)}\right) \in D(M ; Z)$ на множестве $A \subseteq M$, обозначим $f(A)$. Множество аргументов $x^{(n)} \in M$, для которых $f\left(x^{(n)}\right)=m$, обозначим $f^{-1}(m)$.

Для каждого $m \in f(M)$ определим функцию $f^{\langle m\rangle}\left(x^{(n)}\right) \in D\left(M ; Z_{2}\right)$ :

$$
f^{\langle m\rangle}\left(x^{(n)}\right)=\left\{\begin{array}{l}
0, f\left(x^{(n)}\right) \neq m, \\
1, f\left(x^{(n)}\right)=m,
\end{array}\right.
$$

так что $f\left(x^{(n)}\right)=\sum_{m \in f(M)} m f^{\langle m\rangle}\left(x^{(n)}\right)$.

Функцию $f\left(x^{(n)}\right) \in D(M ; Z)$, для которой $f(M) \subseteq Z_{m}$, назовём $m$-функцией от п переменных.

Функцией $k$-значной логики от $n$ переменных называется каждое отображение вида $f: Z_{k}^{n} \rightarrow Z_{k}$. При $k=2$ используется термин булевы фуннкции.

$\mathrm{B}$ принятых обозначениях множество $m$-функций от $n$ переменных совпадает с множеством $D\left(M ; Z_{m}\right)$, множество функций $k$-значной логики от $n$ переменных - с множеством $D\left(Z_{k}^{n} ; Z_{k}\right)$, а множество булевых функций от $n$ переменных - с множеством $D\left(Z_{2}^{n} ; Z_{2}\right)$.

1.3. $\Delta$-графы. В вопросах терминологии теории графов мы будем следовать монографии [4]. По принятым там определениям ориентированные графы могут содержать и петли, и параллельные рёбра. Рассматриваемые далее ориентированные графы могут содержать параллельные рёбра, но петель не содержат. 
Смирнов В. Г.

Рассмотрим следующее семейство $\Delta_{n}^{(k)}$ ориентированных помеченных орграфов (см. [3]).

Определение 1.1. Орграф $H$ с множеством вершин $V(H)$ и множеством рёбер $E(H)$ принадлежит семейству $\Delta_{n}^{(k)}$ ориентированных помеченных орграфов при выполнении условий:

a) Множество $V(H)$ разбивается на $n+1$ непересекающихся подмножеств: $V(H)=\bigcup_{i=0}^{n} V_{i}(H)$, где $V_{i}(H)=\left\{v_{j}^{(i)}, j=0, \ldots, h_{i}-1\right\}$, причём $h_{0}=1, h_{i} \geq 1$ для $i=1, \ldots, n$.

b) Входящая степень вершины $v_{0}{ }^{(0)}$ равна нулю. Входящие степени вершин орграфа $H$, отличных от $v_{0}{ }^{(0)}$, положительны.

c) Из каждой вершины $v \in V_{i}(H)$ при $i<n$ исходит не менее одного и не более $k$ рёбер. Рёбра, исходящие из вершин, принадлежащих множеству $V_{i}(H)$, ведут в вершины из множества $V_{i+1}(H)$. Исходящие степени вершин, принадлежащих множеству $V_{n}(H)$, равны нулю.

d) Каждое ребро $e \in E(H)$, исходящее из вершины $v \in V_{i}(H), i<n$, имеет метку $\gamma_{e} \in Z_{k}$. Рёбра, исходящие из одной вершины, имеют разные метки.

Из определения 1.1 следует, что каждый граф $H \in \Delta_{n}^{(k)}$ является ациклическим и корневым с корнем в вершине $v_{0}{ }^{(0)}$.

Для подмножества семейства $\Delta_{n}^{(k)}$, включающего только орграфы, у которых из каждой вершины $v_{j}^{(i)}$ при $i<n$ исходит точно $k$ рёбер, будем использовать обозначение $\Omega_{n}{ }^{(k)}$.

Все рассматриваемые далее орграфы будем называть графами, поскольку в рамках данной работы это не может вызвать недоразумений.

Каждый граф $H$, принадлежащий при некоторых $n, k$ семейству $\Delta_{n}{ }^{(k)}$, назовём $\Delta$-графом. Аналогично каждый $\Delta$-граф $G$, принадлежащий подмножеству $\Omega_{n}{ }^{(k)}$, назовём $\Omega$-графом. Далее предполагается, что $k-$ фиксированное число.

Вершины $v \in V_{i}(H)$ назовём вершинами $i$-го уровня.

Через $h_{i}$ обозначим число вершин $i$-го уровня, так что $|V(H)|=\sum_{i=0}^{n} h_{i}$.

Через $V_{(v)}(H), V^{(v)}(H)$ обозначим соответственно множество начальных вершин ребер, входящих в вершину $v$, и множество конечных вершин ребер, исходящих из вершины $v$.

Через $E_{(v)}(H), E^{(v)}(H)$ соответственно обозначим множества рёбер графа $H$, входящих в вершину $v$ и исходящих из неё.

Рёбра графа $H$, исходящие из вершин уровня $i-1, i=1, \ldots, n$, назовём рёбрами $i$-го уровня и обозначим их множество через $E_{i}(H)$. 
Ребро с меткой $\gamma$, исходящее из вершины $v$, будем обозначать $(v, \gamma)$. На множестве $E(H)$ определим функцию $\tau$, положив $\tau(v, \gamma)=u$, если ребро $(v, \gamma)$ ведёт в вершину $u \in V(H)$.

Для ребра, соединяющего вершины $v$ и $u$, будем использовать также обозначение $(v, u)$.

1.4. Пути в $\Delta$-графе. Если путь $T$ в $\Delta$-графе $H$ содержит вершину $v$ или ребро $e$, то будем писать $v \in T, e \in T$. В силу ацикличности графа $H$ ориентация рёбер задает естественную частичную упорядоченность множества $V(H) \cup E(H)$. Например, $\left(v_{j}^{(i)}, \gamma\right)<v_{m}{ }^{(l)}$ тогда и только тогда, когда $i<l$ и найдётся путь $T$, для которого $\left(v_{j}^{(i)}, \eta\right), v_{m}{ }^{(l)} \in T$. Элементы $a, b \in V(H) \cup E(H)$ назовём не сравнимыми, если не существует пути $T$ в графе $H$, содержащего $a, b$.

Максимальным путём в ациклическом графе назовём каждый путь максимально возможной длины. Любой максимальный путь $T$ в графе $H \in \Delta_{n}^{(k)}$ имеет длину $n$.

Множество всех максимальных путей в графе $H$ обозначим $\Theta(H)$.

Из определения 1.1 следует, что для сравнимых элементов $a, b \in V(H) \cup$ $\cup E(H)$ найдётся хотя бы один такой путь $T \in \Theta(H)$, что $a, b \in T$.

1.5. Вершинные и рёберные функции $\Delta$-графа. Определим отображение $\Gamma_{H}: \Theta(H) \rightarrow Z_{k}^{n}$, при котором пути $T \in \Theta(H)$ соответствует $n$-последовательность $\gamma^{(n)}=\left(\gamma_{1}, \ldots, \gamma_{n}\right)$ меток принадлежащих ему рёбер.

Образ множества $\Theta(H)$ при отображении $\Gamma_{H}$ обозначим $M(H)$.

Очевидно, что отображение $\Gamma_{H}: \Theta(H) \rightarrow M(H)$ является взаимно однозначным. Отображение, обратное к $\Gamma_{H}$, обозначим $\Gamma_{H}^{-1}$.

Используя отображение $\Gamma_{H}{ }^{-1}$, каждому ребру $e \in E(H)$ и каждой вершине $v \in V(H)$ сопоставим функции $\varphi_{e}^{H}\left(x^{(n)}\right), \psi_{v}^{H}\left(x^{(n)}\right) \in D\left(M(H) ; Z_{2}\right)$ в соответствии со следующим определением.

Определение 1.2. а) Рёберной функиией графа $H$, соответствующей ребру $e \in E(H)$, назовём функцию $\varphi_{e}^{H}\left(x^{(n)}\right)$, задаваемую соотношением

$$
\varphi_{e}^{H}\left(x^{(n)}\right)=\left\{\begin{array}{l}
1, e \in \Gamma_{H}^{-1}\left(x^{(n)}\right), \\
0, e \notin \Gamma_{H}^{-1}\left(x^{(n)}\right) .
\end{array}\right.
$$

b) Вершинной функиией графа $H$, соответствующей вершине $v \in V(H)$, назовём функцию $\psi_{v}^{H}\left(x^{(n)}\right)$, задаваемую соотношением

$$
\psi_{v}^{H}\left(x^{(n)}\right)=\left\{\begin{array}{l}
1, v \in \Gamma_{H}^{-1}\left(x^{(n)}\right), \\
0, v \notin \Gamma_{H}^{-1}\left(x^{(n)}\right) .
\end{array}\right.
$$


Если ясно, о каком графе идёт речь, будем далее опускать указание на него в обозначениях рёберных и вершинных функций.

Из определений 1.1, 1.2 вытекают следующие соотношения:

$$
\begin{gathered}
\sum_{v \in V_{i}(H)} \psi_{v}\left(x^{(n)}\right)=1, i=0, \ldots, n, \\
\sum_{e \in E_{i}(H)} \varphi_{e}\left(x^{(n)}\right)=\mathbf{1}, i=1, \ldots, n, \\
\psi_{v}\left(x^{(n)}\right) \cdot \psi_{u}\left(x^{(n)}\right)=\mathbf{0},
\end{gathered}
$$

если вершины $v, u$ не сравнимы,

$$
\varphi_{e}\left(x^{(n)}\right) \cdot \varphi_{f}\left(x^{(n)}\right)=\mathbf{0},
$$

если рёбра $e, f$ не сравнимы.

Вершинные функции $\Delta$-графа являются линейными комбинациями его рёберных функций:

$$
\begin{aligned}
& \psi_{v}\left(x^{(n)}\right)=\sum_{e \in E_{(v)}} \varphi_{e}\left(x^{(n)}\right), v \in \bigcup_{i=1}^{n} V_{i}(H), \\
& \psi_{v}\left(x^{(n)}\right)=\sum_{e \in E^{(v)}} \varphi_{e}\left(x^{(n)}\right), v \in \bigcup_{i=0}^{n-1} V_{i}(H) .
\end{aligned}
$$

Из (1.1), (1.2) следует, что

$$
\sum_{e \in E_{(v)}} \varphi_{e}\left(x^{(n)}\right)-\sum_{e \in E^{(v)}} \varphi_{e}\left(x^{(n)}\right)=\mathbf{0}, v \in \bigcup_{i=1}^{n-1} V_{i}(H)
$$

Рёберные функции $\Delta$-графа следующим образом связаны с вершинными функциями:

$$
\varphi_{\left(v_{j}^{(i)}, \gamma\right)}\left(x^{(n)}\right)=\left\{\begin{array}{l}
\psi_{v_{j}^{(i)}}\left(x^{(n)}\right) \cdot x_{i+1}^{\langle\gamma\rangle}, \text { если }\left|E^{\left(v_{j}^{(i)}\right)}\right|>1, \\
\psi_{v_{j}^{(i)}}\left(x^{(n)}\right), \text { если }\left|E^{\left(v_{j}^{(i)}\right)}\right|=1,
\end{array}\right.
$$

Из (1.1), (1.2), (1.3) вытекает, что идеал кольца $D(M ; Z)$, порождённый вершинными функциями $\Delta$-графа, совпадает с идеалом, порождённым рёберными функциями.

1.6. H-модули дискретных функций. Множество рёберных функций графа $H \in \Delta_{n}^{(k)}$ порождает модуль над кольцом целых чисел, который обозначим $L_{Z}(H)$. Из определения 1.2 следует, что $L_{Z}(H) \subseteq D(M(H) ; Z)$. 
Аналогично, множество всех линейных комбинаций вершинных функций графа $H \in \Delta_{n}^{(k)}$ с коэффициентами из кольца $Z$ целых чисел образует $Z$-модуль, который обозначим $L_{Z}^{\prime}(H)$. Из соотношений (1.1), (1.2) следует, что модуль $L_{Z}^{\prime}(H)$ является подмодулем модуля $L_{Z}(H)$.

Модули $L_{Z}(H), L_{Z}^{\prime}(H)$ далее будем называть $H$-модулями дискретных функиий.

Так как указанные модули являются модулями без кручения, они имеют базисы. Размерность модуля $L_{Z}(H)$ согласно статье [3] задаётся соотношением

$$
\operatorname{dim} L_{Z}(H)=\sum_{i=0}^{n-1} \sum_{r=2}^{k}(r-1) h_{i}^{(r)}+1
$$

Для указания размерности модуля $L_{Z}^{\prime}(H)$ введём несколько определений (см. [3]).

Множество $N=X \cup Y$ вершин графа $H$ назовём изолированным, если $X \subseteq V_{i}(H), Y \subseteq V_{i+1}(H)$ для некоторого $i, 0 \leq i<n$, и для каждого пути $T \in \Theta(H)$ соотношения $T \cap X \neq \varnothing, T \cap Y \neq \varnothing$ выполняются только одновременно. Изолированное множество вершин графа назовём точно изолированным, если оно не содержит собственных изолированных подмножеств.

Число точно изолированных множеств вершин графа $H$ обозначим $\pi(H)$.

Размерность модуля $L_{Z}^{\prime}(H)$ даётся согласно статье [3] соотношением

$$
\operatorname{dim} L_{Z}^{\prime}(H)=|V(H)|-\pi(H) .
$$

1.7. Максимальный $\Delta$-граф. Рассмотрим граф $G^{(n, k)} \in \Omega_{n}{ }^{(k)}$, содержащий максимально возможное число вершин.

Этот граф является полным $k$-ичным деревом. В каждую вершину $v \in V_{i}\left(G^{(n, k)}\right), i=1, \ldots, n$, входит ровно одно ребро. Поэтому в любую вершину $v$ из корневой вершины ведёт единственный путь, последовательность меток которого обозначим $\Gamma(v)$.

Занумеруем вершины из множества $V_{i}, i=1, \ldots, n$, числами от 0 до $k^{i}-1$ так, чтобы путь $T\left(a_{1}, \ldots, a_{i}\right)$, составленный из рёбер с метками $\left(a_{1}, \ldots, a_{i}\right)$, вёл в вершину $v_{\left[a_{1}, \ldots, a_{i}\right]_{k}}^{(i)}$. При выбранной нумерации вершин имеем $\Gamma\left(v_{\left[a_{1}, \ldots, a_{i}\right]_{k}}^{(i)}\right)=\left(a_{1}, \ldots, a_{i}\right)$.

Ребру $e$, соединяющему вершины $v_{\left[a_{1}, \ldots, a_{n-1}\right]_{k}}^{(n-1)}$ и $v_{\left[a_{1}, \ldots, a_{n}\right]_{k}}^{(n)}$, соответствует функция

$$
\varphi_{e}\left(x^{(n)}\right)=x_{1}^{\left\langle a_{1}\right\rangle} \cdot \ldots \cdot x_{n}^{\left\langle a_{n}\right\rangle} .
$$


Очевидно, что совокупность этих функций составляет базис $Z$-модуля $D\left(Z_{k}^{n} ; Z\right)$. Следовательно, $L_{Z}\left(G^{(n, k)}\right)=D\left(Z_{k}^{n} ; Z\right)$.

Для рассматриваемого графа имеем

$$
\operatorname{dim} L_{Z}\left(G^{(n, k)}\right)=\operatorname{dim} M_{Z}\left(G^{(n, k)}\right)=k^{n} .
$$

1.8. Отображения $\Delta$-графов. Дадим определение отображений, при которых образом графа $H \in \Delta_{n}^{(k)}$ является некоторый граф $F \in \Delta_{n}^{(m)}, m \leq k$.

Определение 1.3. Граф $G \in \Delta_{n}^{(m)}$ назовём $(\Lambda, \Phi)$-образом графа $H \in \Delta_{n}^{(m)}$, если существуют сюръективные отображения $\Lambda: V(H) \rightarrow V(G)$ и $\Phi: Z_{k} \rightarrow Z_{m}$, удовлетворяющие условиям:

1) $\Lambda\left(V_{i}(H)\right)=V_{i}(G), i=1, \ldots, n$;

2) если $u, v \in V(H), u=\tau(v, \gamma)$, то $\Lambda(u)=\tau(\Lambda(v), \Phi(\gamma))$.

Если отображения $\Lambda, \Phi$ инъективны, то графы $G, H$ назовём $(\Lambda, \Phi)$-эквивалентными. Если отображение $\Phi$ является тождественным, то граф $G$ назовём $\Lambda$-образом графа $H$.

Докажем следующее утверждение.

Теорема 1.1. Каждый граф $G \in \Delta_{n}^{(m)}$ при условии $m \leq k$ является $(\Lambda$, Ф)-образом графа $G^{(n, k)}$.

Доказательство. 1) Пусть $m<k$. Покажем, что граф $G^{(n, m)}$ является $(\Lambda, \Phi)$-образом графа $G^{(n, k)}$. Зададим любое сюръективное отображение $\Phi: Z_{k} \rightarrow Z_{m}$. Если в вершину $v \in V_{i}\left(G^{(n, k)}\right)$ ведёт путь с последовательностью меток $\Gamma(v)=\left(\gamma_{1}(v), \ldots, \gamma_{i}(v)\right)$, то положим $\Lambda(v)=v^{\prime}$, где $v^{\prime} \in V\left(G^{(n, m)}\right)$ и $\Gamma\left(v^{\prime}\right)=$ $=\left(\Phi\left(\gamma_{1}(v)\right), \ldots, \Phi\left(\gamma_{i}(v)\right)\right)$.

Нетрудно убедиться, что построенная пара отображений $\Lambda$, Ф удовлетворяет условиям определения 1.3.

2) Пусть $m=k$. Покажем, что любой граф $G \in \Delta_{n}^{(k)}$ является $\Lambda$-образом графа $G^{(n, k)}$. Множество последовательностей меток путей в графе $G$, ведущих из корня в вершину $v^{\prime}$, обозначим $M\left(v^{\prime}\right)$. Отображение $\Lambda: V\left(G^{(n, k)}\right) \rightarrow V(H)$ зададим соотношением

$$
\Lambda(v)=v^{\prime} \Leftrightarrow \Gamma(v) \in M\left(v^{\prime}\right) .
$$

Легко проверить, что пара отображений $\Lambda, \Phi$, где $\Phi-$ тождественное отображение, удовлетворяет условиям определения 1.3. Теорема доказана.

Теорема 1.2. Если граф $G$ является $\Lambda$-образом графа $H$, то модуль $L_{Z}(G)$ изоморфен подмодулю модуля $L_{Z}(H)$. 
Доказательство. Отображение $\Lambda: V(H) \rightarrow V(G)$ индуцирует отображение $\Lambda^{\prime}: E(H) \rightarrow E(G)$, задаваемое соотношением $\Lambda^{\prime}(v, u)=(\Lambda(v), \Lambda(u))$. Следовательно, для $r \in E(G), e \in E(H)$ имеем

$$
\varphi_{r}^{G}\left(x^{(n)}\right)=\sum_{e \in \Lambda^{-1}(r)} \varphi_{e}^{H}\left(x^{(n)}\right),
$$

что доказывает утверждение теоремы.

1.9. Н-функции. Функции $f\left(x^{(n)}\right) \in L_{Z}(H)$ назовём $H$-функциями. Функции из множества $D\left(M(H) ; Z_{m}\right) \cap L_{Z}(H), m \geq 2$, назовём $(H, m)$-функииями. По определению, если $l>m$, то $(H, m)$-функция является также $(H, l)$ функцией.

Если $H \in \Omega_{n}{ }^{(k)}$, то $(H, k)$-функции являются функциями $k$-значной логики.

Каждая $H$-функция $f\left(x^{(n)}\right)$ может быть по определению задана коэффициентами линейной формы над кольцом $Z$ :

$$
f\left(x^{(n)}\right)=\sum_{e \in E(H)} c_{e}^{f} \cdot \phi_{e}\left(x^{(n)}\right),
$$

где $x^{(n)} \in M(H)$, причем такое задание не является единственным. Любое выражение (1.4) назовём $H$-представлением функции $f\left(x^{(n)}\right)$.

Каждое $H$-представление (1.4) функции $f\left(x^{(n)}\right)$ задаёт отображение $c^{(f)}: E(H) \rightarrow Z$ множества ребер графа $H$ в кольцо $Z$, при котором $c^{(f)}(e)=c_{e}^{f}$. Величину $c_{e}^{f}$ назовем весом ребра е при отображении $c^{(f)}$. Весом $\omega\left(c^{(f)}, T\right)$ непустого пути $T$ в графе $H$ назовем сумму весов принадлежащих $T$ ребер:

$$
\omega\left(c^{(f)}, T\right)=\sum_{e \in T} c_{e}^{f} .
$$

Для пустого пути $T$ положим по определению $\omega\left(c^{(f)}, T\right)=0$.

Из принятых определений следует, что при $T \in \Theta(H)$ для любого отображения $c^{(f)}$ справедливо равенство $\omega\left(c^{(f)}, T\right)=f\left(\Gamma_{H}(T)\right)$. Поэтому вес каждого максимального пути графа $H$ не зависит от выбора отображения $c^{(f)}$, и естественно положить его равным $\omega_{f}(T)$. Следовательно, для $x^{(n)} \in M(H)$ верно соотношение $f\left(x^{(n)}\right)=\omega_{f}\left(\Gamma_{H}^{-1}\left(x^{(n)}\right)\right)$.

Нормализованной формой $H$-функции $f\left(x_{n-1}^{(n)}\right)$ назовём такое её $H$-представление, в котором для каждой вершины $v \in \bigcup_{i=1}^{n-1} V_{i}$ выполняется условие

$$
\min _{e \in E^{(v)}} c_{e}^{f}=0 .
$$

В статье [3] показано, что каждая $H$-функция $f\left(x^{(n)}\right)$ имеет единственную нормализованную форму, причём трудоёмкость её получения из любого $H$-представления функции $f\left(x^{(n)}\right)$ пропорциональна $|V(H)|$. 
Коэффициенты нормализованной формы $H$-функции $f\left(x^{(n)}\right)$ обозначим $n_{e}^{f}$, $e \in E(H)$.

ЗАМЕЧАНИЕ 1.1. Из определения нормализованной формы следуют такие её свойства (см. [1]):

1) $\min _{x^{(n)} \in M(H)} f\left(x^{(n)}\right)=\min _{e \in E_{1}(H)} n_{e}^{f}$;

2) $\min _{x^{(n)} \in M(H)} f\left(x^{(n)}\right) \leq n_{e}^{f} \leq \max _{x^{(n)} \in M(H)} f\left(x^{(n)}\right)$;

$3)$ для каждого коэффициента $n_{e}^{f}$ нормализованной формы $(H, m)$-функции $f\left(x^{(n)}\right)$ выполняется условие $0 \leq n_{e}^{f} \leq m-1$;

$4)$ нормализованная форма $(H, 2)$-функции имеет вид

$$
f\left(x^{(n)}\right)=\sum_{e \in W} n_{e}^{f} \varphi_{e}\left(x^{(n)}\right),
$$

где $W$ - множество попарно не сравнимых рёбер графа $H$.

1.10. Ортогонально разложимые $\boldsymbol{H}$-функции.

Определение 1.4. Ортогонально разложимой Н-функиией назовём $H$-функцию $f\left(x^{(n)}\right)$, для которой существует $H$-представление

$$
f\left(x^{(n)}\right)=\sum_{e \in W} c_{e}^{f} \varphi_{e}\left(x^{(n)}\right),
$$

где $W$ - множество попарно не сравнимых рёбер графа $H$.

Из свойства 4) замечания 1.1 следует, что каждая $(H, 2)$-функция ортогонально разложима.

Теорема 1.3. Функиия $f\left(x^{(n)}\right) \in D(M(H) ; Z)$ является ортогонально разложимой Н-функиией тогда и только тогда, когда функции $f^{\langle m\rangle}\left(x^{(n)}\right)$, $m \in f(M(H))$, являются Н-функциями.

Доказательство. Допустим, что функции $f^{\langle m\rangle}\left(x^{(n)}\right), m \in f(M(H))$, являются $H$-функциями. Так как $(H, 2)$-функции ортогонально разложимы, имеем

$$
f^{\langle m\rangle}\left(x^{(n)}\right)=\sum_{e \in W_{m}} \varphi_{e}\left(x^{(n)}\right),
$$

где множества рёбер $W_{m}, m \in f(M(H))$, состоят из попарно не сравнимых рёбер графа $H$. В силу попарной ортогональности функций $f^{\langle m\rangle}\left(x^{(n)}\right)$ множе- 
ство $W=\underset{m \in M(H)}{\cup} W_{m}$ также состоит из попарно не сравнимых рёбер. Следовательно, для $H$-функции $f\left(x^{(n)}\right)$ верно соотношение (1.5), где при $e \in W_{m}$ имеем $c_{e}^{f}=m$.

Таким образом, $f\left(x^{(n)}\right)$ - ортогонально разложимая $H$-функция.

Обратно, пусть функция $f\left(x^{(n)}\right)$ является $H$-ортогонально разложимой $H$-функцией и может быть представлена в виде (1.5). Для каждого $m \in f(M(H))$ построим множество $W_{m} \subseteq W$, положив $e \in W_{m}$, если $c_{e}^{f}=m$. Очевидно, что

$$
f^{\langle m\rangle}\left(x^{(n)}\right)=\sum_{e \in W_{m}} \varphi_{e}\left(x^{(n)}\right),
$$

т. е. функции $f^{\langle m\rangle}\left(x^{(n)}\right), m \in f(M(H))$, являются $H$-функциями. Теорема доказана.

Теорема 1.4. Если Н-функиия $f\left(x^{(n)}\right)$ ортогонально разложима, то при любых $m, a \in Z, m>1$, функция $g\left(x^{(n)}\right)=f\left(x^{(n)}\right)+\underset{m}{+}$ а является ортогонально разложимой Н-функиией.

Доказательство. Пусть $f(M(H))=\left\{d_{1}, \ldots, d_{h}\right\}$, тогда $g(M(H))=\left\{d_{1}+\underset{m}{+}\right.$ $\left.+a, \ldots, \quad d_{h} \underset{m}{+} a\right\}$. Из определения функции $g\left(x^{(n)}\right)$ следует, что $g^{<d_{i}+a>}\left(x^{(n)}\right) \equiv f^{<d_{i}>}\left(x^{(n)}\right)$. Отсюда и из теоремы 1.3 следует справедливость утверждения теоремы.

Далее ортогонально разложимую $H$-функцию $f\left(x^{(n)}\right)$ будем называть $H_{0}$-функиией. Аналогично, ортогонально разложимую $(H, m)$-функцию $f\left(x^{(n)}\right)$ будем называть $(H, m)_{0}$-функиией.

\section{2. Алгоритмы решения $\boldsymbol{H}$-систем дискретных уравнений}

2.1. Н-системы дискретных уравнений. Согласно п. 1.6 с каждым $\Delta$-графом $H$ связан $H$-модуль дискретных функций. Рассмотрим системы дискретных уравнений, левые части которых являются $H$-функциями, где $H \in \Omega_{n}{ }^{(k)}$.

Определение 2.1. 1) Систему дискретных уравнений

$$
f_{j}\left(x^{(n)}\right)=a_{j}, j=1, \ldots, t,
$$

где $f_{j}\left(x^{(n)}\right) \in L_{Z}(H), H \in \Omega_{n}{ }^{(k)}, a_{j} \in Z, j=1, \ldots, t$, назовём $H$-системой.

2) Назовём Н-уравнением $H$-систему вида (2.1), для которой $t=1$. 
3) Назовём $H$-систему дискретных уравнений (2.1) ортогонально разложимой Н-системой, или $H_{0}$-системоц̆, если функции $f_{j}\left(x^{(n)}\right), j=1, \ldots, t$, ортогонально разложимы.

4) $H$-систему дискретных уравнений (2.1), где $f_{j}(M(H)) \subseteq Z_{m}, a_{j} \in Z_{m}$, $j=1, \ldots, t$, назовём $(H, m)$-системой. Если при этом система (2.1) является $H_{0}$-системой, то назовём её $(H, m)_{0}$-системой.

Из свойства 4 замечания 1.1 следует, что каждая $(H, 2)$-система ортогонально разложима.

Множество решений системы уравнений $S$ обозначим sol( $S)$. Множество решений уравнения $f\left(x^{(n)}\right)=0$ обозначим $\operatorname{sol}(f)$.

Для произвольной $H$-функции $f\left(x^{(n)}\right)$ положим

$$
r(f)=\max _{x^{(n)} \in M(H)} f\left(x^{(n)}\right)-\min _{x^{(n)} \in M(H)} f\left(x^{(n)}\right) .
$$

Для $H$-системы дискретных уравнений $S$, заданной соотношениями (2.1), положим

$$
r(S)=\max _{j=1, \ldots, t} r\left(f_{j}\right) .
$$

Теорема 2.1. Если система дискретных уравнений $S$, заданная соотношениями (2.1), является $H_{0}$-системой, то при произвольных значениях правых частей $a_{j} \in Z_{m}, j=1, \ldots, t$, где $m>r(S)$, система уравнений

$$
f_{j}\left(x^{(n)}\right) \underset{m}{-} a_{j}=0, j=1, \ldots, t
$$

является $H_{0}$-системой, равносильной системе $S$.

Доказательство. С учётом теоремы 1.4 достаточно показать, что при всех $a \in Z_{m}, m>r(f)$ из ортогональной разложимости $H$-уравнения $f\left(x^{(n)}\right)=a$ вытекает его равносильность $H$-уравнению $f\left(x^{(n)}\right)_{m} a=0$. Пусть $f(M(H))=\left\{d_{1}, \ldots, d_{h}\right\}$, так что $r(f)=d_{h}-d_{1}$. Указанная равносильность следует из того факта, что при любых $m>r(f), a \in Z_{m}$ отображение $\left(d_{1}, \ldots, d_{h}\right) \rightarrow$ $\rightarrow\left(d_{1} \underset{m}{+} a, \ldots, d_{h}+\underset{m}{a}\right)$ является взаимно однозначным.

Если условия теоремы нарушены, то система (2.2) не обязательно равносильна системе (2.1).

Следствие 2.1. (H, $k)_{0}$-система уравнений $S$, заданная соотношениями (2.1), равносильна однородной $(H, k)_{0}$-системе уравнений $S^{\prime}$, заданной соотношениями

$$
f_{j}\left(x^{(n)}\right)-a_{j}=0, j=1, \ldots, t .
$$


Утверждение следствия вытекает из того, что для $(H, k)$-системы уравнений $S$ имеем $r(S)<k$.

2.2. $\Delta$-подграфы и графы решений $H$-систем дискретных уравнений. Предложенные в статьях $[1,3]$ алгоритмы решения $H$-систем дискретных уравнений $S$ применимы в тех случаях, когда множество $\Gamma_{H}^{-1}(\operatorname{sol}(S))$ совпадает с множеством путей некоторого подграфа графа $H$.

Определение 2.2. Назовём $\Delta$-подграфом графа $H \in \Delta_{n}{ }^{(k)}$ каждый его подграф, являющийся объединением непустого множества путей $T \in \Theta(H)$, причём рёбра подграфа сохраняют присвоенные им метки.

Из определений 2.2 и 1.1 следует, что каждый $\Delta$-подграф графа $H \in \Delta_{n}^{(k)}$ принадлежит семейству $\Delta_{n}^{(k)}$.

Непустому множеству $A \subseteq M(H)$ поставим в соответствие следующий $\Delta$-подграф графа $H \in \Delta_{n}^{(k)}$ :

$$
H[A]=\underset{a^{(n)} \in A}{\cup} \Gamma_{H}^{-1}\left(a^{(n)}\right) .
$$

Граф $H[A]$ может содержать, вообще говоря, максимальные пути, не принадлежащие множеству $A$. Выделим случаи, когда этого не происходит, и назовём $H$-замкнутым множеством непустое множество $B \subseteq M(H)$, для которого $B=M(H[B])$.

ЗАМЕЧАНИЕ 2.1. В статье [1] (см. лемму 4.3 и следствие 4.1) показано:

1) непустое пересечение $H$-замкнутых множеств $H$-замкнуто;

2) для каждой $H$-функции $f\left(x^{(n)}\right)$ множества

$$
M_{f}=\left\{x^{(n)} \mid f\left(x^{(n)}\right)=\min _{x^{(n)} \in M(H)} f\left(x^{(n)}\right)\right\}, M^{f}=\left\{x^{(n)} \mid f\left(x^{(n)}\right)=\max _{x^{(n)} \in M(H)} f\left(x^{(n)}\right)\right\}
$$

являются $H$-замкнутыми множествами.

Определение 2.3. Графом решений $H$-системы $S$ дискретных уравнений назовём $\Delta$-подграф $G(S)$ графа $H$, удовлетворяющий условию $M(G(S))=\operatorname{sol}(S)$.

Из определения 2.3 следует, что граф решений совместной $H$-системы $S$ существует тогда и только тогда, когда $H$-замкнуто множество $\operatorname{sol}(S)$. В этом случае $G(S)=H[\operatorname{sol}(S)]$.

Для $H$-системы $S$ положим

$$
G=\underset{\gamma^{(n)} \in \operatorname{sol}(S)}{\cup} \Gamma_{H}^{-1}\left(\gamma^{(n)}\right) .
$$

Теорема 2.2. Граф $G$ является графом решений совместной однородной $(H, k)$-системы $S$ дискретных уравнений.

Доказательство. Пусть $(H, k)$-система $S$ задана соотношениями (2.1), где $a_{j}=0, j=1, \ldots, t$. Из очевидного соотношения

$$
\operatorname{sol}(S)=\overbrace{j=1}^{t} \operatorname{sol}\left(f_{j}\right)
$$


Смирнов В. Г.

и замечания 2.1 вытекает $H$-замкнутость множества $\operatorname{sol}(S)$, следовательно, $M(G(S))=\operatorname{sol}(S)$, что доказывает теорему.

Следствие 2.2. Граф решений совместной $(H, k)_{0}$-системы $S$ существует.

Действительно, утверждение следствия вытекает из следствия 2.1 и теоремы 2.2.

2.3. Построение $(H, 2)$-системы, равносильной $(H, k)_{0}$-системе. В отличие от алгоритма построения графа решений из статьи [3], где используется переход к равносильной однородной $(H, k)$-системе, предлагаемый далее в п. 2.4 алгоритм основан на переходе к равносильной $(H, 2)$-системе, что, как показано в п. 2.5, расширяет границы его применимости.

Зададим отображение $\delta: D(M(H) ; Z) \rightarrow D\left(M(H) ; Z_{2}\right)$, положив для $f\left(x^{(n)}\right) \in D(M(H) ; Z)$

$$
\delta\left(f\left(x^{(n)}\right)\right)=\left\{\begin{array}{l}
0, f\left(x^{(n)}\right)=0 \\
1, f\left(x^{(n)}\right) \neq 0,
\end{array}\right.
$$

и рассмотрим систему уравнений $\delta(S)$, заданную соотношениями

$$
\delta\left(f_{j}\left(x^{(n)}\right)-a_{j}\right)=0, j=1, \ldots, t .
$$

Верно следующее утверждение.

Теорема 2.3. Если система $S$ является $(H, k)_{0}-$ системой, то система $\delta(S)$ является $(H, 2)$-системой, равносильной системе $S$ и Н-уравнению

$$
\sigma_{\delta(S)}\left(x^{(n)} ; a^{(t)}\right)=0
$$

где $\sigma_{\delta(S)}\left(x^{(n)} ; a^{(t)}\right)=\sum_{j=1}^{t} \delta\left(f_{j}\left(x^{(n)}\right)-a_{j}\right)$.

Доказательство. Нетрудно показать, учитывая замечание 1.1, что применение оператора $\delta$ к нормализованной форме $(H, k)_{0}$-функции сводится к замене ненулевых коэффициентов её нормализованной формы на 1, следовательно, система $\delta(S)$ является $(H, 2)$-системой. Равносильность систем уравнений $S, \delta(S)$ и уравнения (2.4) очевидна.

2.4. Алгоритм построения графа решений совместной $(H, k)_{0}$-системы дискретных уравнений. Исходим из задания $(H, k)_{0}$-системы $S$ дискретных уравнений соотношениями (2.1), где функции в левых частях заданы $H$-представлениями.

Алгоритм построения графа решений $(H, k)_{0}$-системы дискретных уравнений реализуется следующей последовательностью шагов.

Алгоритм 2.1. Предварительный этап. Вычислим нормализованные формы функций $f_{j}\left(x^{(n)}\right)-a_{k}, j=1, \ldots, t$. Применим к каждой нормализованной 
форме оператор $\delta$ и получим $(H, 2)$-систему (2.4). Вычислим функцию невязки (2.5) и её нормализованную форму

$$
\sigma_{\delta(S)}\left(x^{(n)} ; a^{(t)}\right)=\sum_{e \in E(H)} n_{e}^{\sigma} \varphi_{e}\left(x^{(n)}\right) .
$$

Получим и запомним множество $E(\sigma)$ таких рёбер графа $H$, не принадлежащих первому уровню, что $n_{e}^{\sigma}=0$.

Шаг 1. Положим:

$$
V_{0}(G(S))=v_{0}^{(0)} ; E_{1}(G(S))=\left\{e \in E_{1}(H) \mid n_{e}^{\rho}=0\right\} .
$$

Если $E_{1}(G(S))=\varnothing$, то система $S$ не совместна и работа алгоритма закончена.

Если $E_{1}(G(S)) \neq \varnothing$, то система совместна.

Шаги $i=2, \ldots, n-1$. Последовательно получим множества $V_{i}(G(S))$ вершин и $E_{i+1}(G(S))$ рёбер, $i=1, \ldots, n-1$ :

$$
\begin{gathered}
V_{i}(G(S))=\left\{v \mid E_{(v)} \cap E_{i}(G(S)) \neq \varnothing\right\}, \\
E_{i+1}(\mathrm{G}(S))=\left(\underset{v \in V_{i}(G(S))}{\cup} E^{(v)}\right) \cap E(\sigma) .
\end{gathered}
$$

Шаг $n$. Получим множество $V_{n}(G(S))$ :

$$
V_{n}(G(S))=\left\{v \mid E_{(v)} \cap E_{n-1}(G(S)) \neq \varnothing\right\} .
$$

Результатом работы алгоритма является граф $G(S)$ с множеством вершин $V(G(S))=\cup_{i=0}^{n} V_{i}(G(S))$ и множеством рёбер $E(G(S))=\cup_{i=0}^{n-1} E_{i}(G(S))$, который по построению является графом решений системы (2.1).

Вычислительная и ёмкостная сложности алгоритма 2.1 по аналогии с теоремой 3.2 из статьи [3] оцениваются при $n, t \rightarrow \infty$ величиной $C \cdot t \cdot|V(H)|$ условных операций, где $C$ - некоторая константа.

По графу решений системы можно перечислить без повторений все её решения. Каждый шаг алгоритма перечисления решений имеет трудоёмкость, пропорциональную числу $n$ неизвестных системы (см. [3]).

2.5. Н-системы с искажённой правой частью. Возможность находить истинные решения системы дискретных уравнений при наличии искажений в правой части существенно расширяет возможности практического применения соответствующего метода.

Рассмотрим следующую вероятностную модель $(H, k)_{0}$-систем дискретных уравнений с искажённой правой частью. $(H, k)_{0}$-систему уравнений $S$, 
заданную соотношениями (2.1), назовём исходной системой. Наряду с ней рассмотрим систему $R$, заданную соотношениями

$$
f_{j}\left(x^{(n)}\right)=b_{j}, j=1, \ldots, t .
$$

Определение 2.4. 1) $(H, k)_{0}$-систему дискретных уравнений $R$, заданную соотношениями (2.6), назовём системой уравнений с искажённой правой частью, если $b_{j}=a_{j}+\varepsilon_{j}$, где $\varepsilon_{j} \in Z_{k}-$ независимые, одинаково распределённые в совокупности случайные величины, $j=1, \ldots, t$.

2) $(H, k)_{0}$-систему дискретных уравнений $\mathrm{c}$ искажённой правой частью $R$, заданную соотношениями (2.6), назовём заведомо совместной, если совместна исходная система уравнений (2.1). Решения исходной системы (2.1) назовём истинными решениями системы (2.6).

Отметим, что исходная $(H, k)_{0}$-система $S$ может рассматриваться как частный случай $(H, k)_{0}$-системы с искажённой правой частью, для которого $P\left(\varepsilon_{j}>0\right)=0$.

Предположим, что $P\left(\varepsilon_{j}=0\right)>P\left(\varepsilon_{j}>0\right)$ и все вероятности $P\left(\varepsilon_{j}=c\right), c=1, \ldots$, $l-1$, равны между собой.

Система уравнений $R$, заданная соотношениями (2.6) согласно теореме 2.3 равносильна $H$-функции

$$
\sigma_{\delta(S)}\left(x^{(n)} ; b^{(t)}\right)=\sum_{j=1}^{t} \delta\left(f_{j}\left(x^{(n)}\right)-b_{j}\right),
$$

которую назовём невязкой системы $R$.

Аналогично §5 статьи [5], где рассматривались системы булевых уравнений с искажённой правой частью, можно показать, что при достаточно широких предположениях векторы $m^{(n)} \in M_{\sigma_{\delta(s)}}$, минимизирующие значение невязки (2.7), являются оценками максимального правдоподобия истинных решений заведомо совместной системы (2.1).

Для нахождения множества $M_{\sigma_{\delta(s)}}$ достаточно внести изменение в алгоритм 2.1, сформулировав шаг 1 в следующем виде.

Шаг 1'. Положим:

$$
V_{0}(G(S))=v_{0}{ }^{(0)} ; E_{1}(G(S))=\left\{e \in E_{1}(H) \mid n_{e}^{\rho}=\min _{r \in E_{1}(H)} n_{r}^{\sigma}\right\} .
$$

Результатом работы модифицированного алгоритма будет граф $G(R) \in \Delta_{n}^{(k)}$, для которого $M(G(R))=M_{\sigma_{\delta(S)}}$.

Вычислительная и ёмкостная сложности алгоритма 2.1 при этом не меняются. 
Комплекс вопросов, связанных с оценками вероятности принадлежности истинных решений заведомо совместной системы уравнений $S$ множеству $M(G(R))$, требует отдельного исследования и остаётся за рамками данной работы.

\section{3. Модули квазисимметрических функций и системы квазисимметрических уравнений}

3.1. $\Delta$-граф $Q$ ?' Рассмотрим граф $Q^{\prime} \in \Omega_{n}{ }^{(k)}$ с множеством вершин

$$
V\left(Q^{\prime}\right)=\left\{v_{j}^{(i)}, i=0, \ldots, n, j=0, \ldots,(k-1) i\right\}
$$

и множеством рёбер

$$
E\left(Q^{\prime}\right)=\left\{\left(v_{j}^{(i)}, \gamma\right), i=0, \ldots, n, j=0, \ldots,(k-1) i, \gamma=0, \ldots,(k-1)\right\},
$$

задаваемых соотношениями

$$
\tau\left(v_{j}^{(i)}, \gamma\right)=v_{j+\gamma}^{(i+1)}, i=0, \ldots, n-1, j=0, \ldots,(k-1) i .
$$

Введём особые обозначения для вершинных и рёберных функций графа $Q^{\prime}$, положив

$$
\psi_{v_{j}^{(i)}}^{\left(Q^{\prime}\right)}\left(x^{(n)}\right)=q_{i, j}^{\prime}\left(x^{(n)}\right), \varphi_{\left(v_{j}^{(i)}, \gamma\right)}^{Q^{\prime}}=e_{i, j, \gamma}^{\prime}\left(x^{(n)}\right) .
$$

Из определения графа $Q^{\prime}$ вытекает, что

$$
q_{0,0}^{\prime}=1, q_{i, j}^{\prime}\left(x^{(n)}\right)=1 \Leftrightarrow x_{1}+\ldots+x_{i}=j, i=1, \ldots, n, j=0, \ldots, i(k-1) .
$$

Отметим, что функции $q_{i, j}^{\prime}\left(x^{(n)}\right), i>0$, являются симметрическими функциями переменных $x_{1}, \ldots, x_{i}$ и

$$
e_{i, j, \gamma}^{\prime}\left(x^{(n)}\right)=q_{i-1, j}^{\prime}\left(x^{(n)}\right) \cdot x_{i}^{\langle\gamma\rangle}, i=1, \ldots, n .
$$

Приведём основные характеристики графа $Q^{\prime} \in \Delta_{n}^{(k)}$ :

$$
\begin{aligned}
& \text { a) } h_{i}\left(Q^{\prime}\right)=(k-1) i+1, i=0, \ldots, n ; \\
& \text { b) } \left.\left|V\left(Q^{\prime}\right)\right|=(k-1) \frac{n(n+1)}{2}+n+1\right) ; \\
& \text { c) }\left|E\left(Q^{\prime}\right)\right|=k(k-1) \frac{n(n-1)}{2}+k n ; \\
& \text { d) } \pi\left(Q^{\prime}\right)=n ; \\
& \text { e) } \operatorname{dim} L_{Z}\left(Q^{\prime}\right)=(k-1)\left((k-1) \frac{n(n-1)}{2}+k-1\right) ; \\
& \text { f) } \operatorname{dim} M_{Z}\left(Q^{\prime}\right)=(k-1) \frac{n(n+1)}{2}+1 .
\end{aligned}
$$


3.2. Свойства модуля $M_{Z}\left(Q^{\prime}\right)$. Как было отмечено в п. 1.6, модуль $M_{Z}\left(Q^{\prime}\right)$ является подмодулем модуля $L_{Z}\left(Q^{\prime}\right)$. Из соотношений $e$ ), $f$ ) следует, что равенство $\operatorname{dim} L_{Z}\left(Q^{\prime}\right)=\operatorname{dim} M_{Z}\left(Q^{\prime}\right)$ имеет место только при $k=2$.

Модуль $M_{Z}\left(Q^{\prime}\right)$ допускает любопытную алгебраическую интерпретацию.

Вектору $a^{(n)} \in Z_{k}^{n}$ поставим в соответствие целочисленный вектор

$$
\theta^{n)}\left(a^{(n)}\right)=\left(\theta_{1}, \ldots, \theta_{n}\right),
$$

где $\theta_{i}=a_{1}+\ldots+a_{i}, i=1, \ldots, n$.

Зададим частичную упорядоченность на множестве $Z_{k}^{n}$, положив $a^{(n)}<b^{(n)}$, если для некоторого $i=1, \ldots, n$ имеем $\theta_{i}\left(a^{(n)}\right)<\theta_{i}\left(b^{(n)}\right)$, но не существует такого значения $j$, что $\theta_{j}\left(a^{(n)}\right)>\theta_{j}\left(b^{(n)}\right)$. Несложно показать, что введённая частичная упорядоченность превращает множество $Z_{k}^{n}$ в дистрибутивную структуру, которую обозначим $D_{n}{ }^{(k)}$.

Объединением векторов $a^{(n)}, b^{(n)}$ в структуре $D_{n}^{\left({ }^{(k)}\right.}$ будет вектор $c^{(n)}$, для которого $\theta_{i}\left(c^{(n)}\right)=\max \left(\theta_{i}\left(a^{(n)}\right), \theta_{i}\left(b^{(n)}\right)\right)$, а их пересечением - вектор $d^{(n)}$, удовлетворяющий условию $\theta_{i}\left(d^{(n)}\right)=\min \left(\theta_{i}\left(a^{(n)}\right), \theta_{i}\left(b^{(n)}\right)\right), i=1, \ldots, n$.

Неразложимыми в объединение элементами структуры $D_{n}{ }^{(k)}$ являются векторы следующего вида:

$$
a^{(n)}=(\underbrace{0, \ldots, 0,}_{i_{1}} a, \underbrace{k-1, \ldots, k-1}_{i_{2}} \underbrace{0, \ldots, 0}_{i_{3}}),
$$

где $a \in Z_{k}, i_{1}+i_{2}+i_{3}=n-1$. Число неразложимых в объединение элементов равно $(k-1) \frac{n(n+1)}{2}+1$.

Функцией оценки на структуре $D(n, k)$ назовём функцию $h\left(x^{(n)}\right) \in D\left(Z_{k}^{n} ; Z\right)$ со свойством:

$$
h\left(a^{(n)} \cup b^{(n)}\right)+h\left(a^{(n)} \cap b^{(n)}\right)=h\left(a^{(n)}\right)+h\left(b^{(n)}\right) .
$$

Известно (см. монографию [6]), что функции оценки на конечной дистрибутивной структуре образуют $Z$-модуль, размерность которого равна числу неразложимых в объединение элементов структуры.

Справедливо следующее утверждение.

Tеорема 3.1. Z-модуль $M_{Z}\left(Q^{\prime}\right)$ совпадает с Z-модулем функций оценки на структуре $D_{n}{ }^{(k)}$.

Доказательство теоремы следует из того факта, что вершинные функции графа $Q^{\prime}$ являются функциями оценки на структуре $D_{n}{ }^{(k)}$, и из равенства размерностей, упомянутых в формулировке теоремы модулей.

3.3. Квазисимметрический граф. Спецификацией $k$-значной $n$-последовательности $x^{(n)}=\left(x_{1}, \ldots, x_{n}\right)$ при $n \geq 1$ называют целочисленный вектор 
$s^{(n)}\left(x^{(n)}\right)=\left(s_{0}, \ldots, s_{k-1}\right)$, где компонента $s_{m}, m=0, \ldots, k-1$, равна числу вхождений числа $m$ в последовательность $x^{(n)}$. Известно (см. [7]), что число спецификаций $k$-значных $n$-последовательностей равно $C_{k-1}^{n+k-1}$.

Занумеруем некоторым образом все возможные векторы спецификаций $k$-значных $i$-последовательностей и обозначим $s^{(i, j)}=\left(s_{0}^{(i, j)}, \ldots, s_{k-1}^{(i, j)}\right), j=1, \ldots$, $C_{k-1}^{i+k-1}, i=1, \ldots, n$. Положим также $s_{0}^{(0)}=(0, \ldots, 0)$.

Рассмотрим граф $Q \in \Omega_{n}{ }^{(k)}$, у которого вершинами уровня $i \geq 0$ являются все возможные спецификации $k$-значных $i$-последовательностей. При $i<n$ из вершины $s^{(i, j)}$ исходит $k$ рёбер с метками $0, \ldots, k-1$ и $\tau\left(s^{(i, j)}, \gamma\right)=s^{(i+1, l)}=$ $=\left(s_{0}^{(i+1, l)}, \ldots, s_{k-1}^{(i+1, l)}\right)$, где $s_{\gamma}^{(i+1, l)}=s_{\gamma}^{(i, l)}+1$, а при $\eta \neq \gamma$ имеем $s_{\eta}^{(i+1, l)}=s_{\eta}^{(i, l)}$.

Отметим, что при $k=2$ графы $Q$ и $Q^{\prime}$ совпадают.

Введём особые обозначения для вершинных и рёберных функций графа $Q$, положив

$$
\psi_{v_{j}^{(i)}}^{(Q)}\left(x^{(n)}\right)=q_{i, j}\left(x^{(n)}\right), \varphi_{\left(v_{j}^{(i)}, \gamma\right)}^{Q}=e_{i, j, \gamma}\left(x^{(n)}\right) .
$$

Множество $i$-последовательностей $x^{(i)}$ со специализацией $s^{(i, j)}$ обозначим $\left\{s^{(i, j)}\right\}$.

Из определения графа $Q$ следует, что его вершинные и рёберные функции задаются соотношениями

$$
\begin{gathered}
q_{0,0}=1, q_{i, j}\left(x^{(i)}\right)=1 \Leftrightarrow=x^{(i)} \in\left\{s^{(i, j)}\right\}, i=1, \ldots, n, j=1, \ldots, C_{k-1}^{i+k-1}, \\
e_{i, j, \gamma}\left(x^{(n)}\right)=q_{i, j}\left(x^{(n)}\right) \cdot x_{i+1}^{\langle(\gamma)}, i=0, \ldots, n-1 .
\end{gathered}
$$

Легко убедиться, что граф $Q^{\prime}$ является $\Lambda$-образом графа $Q$, где отображение $\Lambda$ задаётся соотношением

$$
\Lambda\left(s^{(i, j)}\right)=v_{m}^{(i)} \leftrightarrow \sum_{l=0}^{k-1} l \cdot s_{l}^{(i, j)}=m .
$$

В соответствии с теоремой 1.2 модуль $L_{Z}\left(Q^{\prime}\right)$ изоморфен некоторому подмодулю модуля $L_{Z}(Q)$. В дальнейшем будем отождествлять модуль $L_{Z}\left(Q^{\prime}\right)$ и изоморфный ему подмодуль модуля $L_{Z}(Q)$.

Приведём основные характеристики графа $Q$ и модуля $L_{Z}(Q)$ :

$$
\begin{aligned}
& \text { a) } h_{i}(Q)=C_{k-1}^{i+k-1}, i=0, \ldots, n \\
& \text { b) }|V(Q)|=\sum_{i=0}^{n} C_{i+k-1}^{k-1} ; \\
& \text { c) }|E(Q)|=k \sum_{i=0}^{n-1} C_{i+k-1}^{k-1}
\end{aligned}
$$




$$
\begin{aligned}
& \text { d) } \pi(Q)=n ; \\
& \text { e) } \operatorname{dim} L_{Z}(Q)=(k-1) \sum_{i=0}^{n-1} C_{k-1}^{i+k-1}+1 ; \\
& \text { f) } \operatorname{dim} M_{Z}(Q)=\sum_{i=0}^{n} C_{k-1}^{i+k-1}-n .
\end{aligned}
$$

Функции $f\left(x^{(n)}\right) \in L_{Z}(Q)$ назовём квазисимметрическими функциями. Граф $Q$ назовём квазисимметрическим графом, а модуль $L_{Z}(Q)$ и его подмодули - модулями квазисимметрических функций. Таким образом, каждая квазисимметрическая функция может быть задана линейной комбинацией рёберных функций модуля $L_{Z}(Q)$ :

$$
f\left(x^{(n)}\right)=\sum_{\left(v_{j}^{(i)}, \gamma\right) \in E\left(Q^{\prime}\right)} c_{\left(v_{j}^{(i)}, \gamma\right)}^{f} \cdot e_{i, j, \gamma}\left(x^{(n)}\right) .
$$

3.4 Метод решения систем квазисимметрических уравнений. Систему уравнений $S$, заданную соотношениями (2.1), назовём системой квазисимметрических уравнений, если $f_{j}\left(x^{(n)}\right) \in L_{Z}(Q), j=1, \ldots, t$.

Обозначим через $S(Q, m, n, t)$ и $S\left(Q^{\prime}, m, n, t\right)$ множества $(Q, m)$-систем и, соответственно, $\left(Q^{\prime}, m\right)$-систем квазисимметрических уравнений, содержащих $n$ неизвестных и состоящих из $t$ уравнений.

Каждая $(Q, m)_{0}$-система квазисимметрических уравнений может быть задана уравнениями вида

$$
f_{j}\left(x^{(n)}\right)=\sum_{\left(v_{s}^{(i)}, \gamma\right) \in W_{j}} c_{\left(v_{s}^{(i)}, \gamma\right)}^{f_{j}} \cdot e_{i, s, \gamma}\left(x^{(n)}\right)=a_{j}, j=1, \ldots, t,
$$

где $W_{j}$ - множества попарно не сравнимых рёбер графа $Q$.

Применение к $(Q, m)_{0}$-системам квазисимметрических уравнений алгоритма 2.1 приводит с учётом параметров графов $Q, Q^{\prime} \in \Delta_{n}^{(k)}$ к следующему утверждению.

Теорема 3.2. При $n, t \rightarrow \infty$ верны следуюшие оченки.

1) Вычислительная и ёмкостная сложности применения алгоритма 2.1 $\kappa$ системе $S \in S(Q, m, n, t)$ очениваются величинами порядка $O\left(t \cdot n^{k}\right)$ условных операций и $O\left(t \cdot n^{k}\right)$ единиц памяти.

2) Вычислительная и ёмкостная сложности применения алгоритма 2.1 $\kappa$ системе $S \in S\left(Q^{\prime}, l, n, t\right)$ очениваются величинами порядка $O\left(t \cdot n^{2}\right)$ условных операчий и $O\left(t \cdot n^{2}\right)$ единиц памяти.

\section{4. Применение квазисимметрических функций при анализе генераторов с управляемым движением}

4.1. Генераторы с управляемым движением. Распространённым способом усложнения криптографических преобразований в схемах на основе регистров с линейной обратной связью (РСЛОС) является использование 
регистров с неравномерным движением информации. Генераторы, использующие такие регистры, обычно называют генераторами с управляемым движением. Для них характерно наличие управляющего регистра и одного или нескольких управляемых или генерирующих регистров. Движение информации в управляющем регистре регулярное, на один шаг за такт. Знак результирующей последовательности зависит от состояний генерирующих регистров. В рамках описанной криптографической схемы возможно использование разнообразных способов управления и получения результирующей последовательности.

Обзор схем генераторов с управляемым движением и методов их анализа можно найти в обзоре [8] и монографии [9]. Из статьи [10] можно сделать вывод, что известные алгебраические атаки на генераторы с управляемым движением над полем GF(2) основаны на опробовании состояний управляющего регистра. Далее будет описан алгебраический метод атаки на генераторы над произвольным простым полем, использующий опробование состояний управляемых регистров, причём допустимы искажения в результирующей последовательности. Какой вариант атаки в случае поля GF(2) эффективнее, зависит от конкретных значений параметров генератора.

Далее рассматриваются генераторы над полем $\mathrm{GF}(k)$, где $k$ - простое число.

4.2. $(\mathbf{1}, \ldots, \boldsymbol{k})$-генератор. Этот генератор содержит управляющий регистр с линейной обратной связью (РСЛОС) $A$ и генерирующий регистр $B$. Состояниями регистров $A, B$ являются векторы над полем $\mathrm{GF}(k)$ размерностей $m_{A}, m_{B}$ соответственно. На состояниях регистра $A$ задана аффинная функция $u\left(x^{\left(m_{A}\right)}\right) \in D\left(\operatorname{GF}(k)^{m_{A}}, Z_{k}\right)$, принимающая значения $1, \ldots, k$. На состояниях регистра $B$ задана функция $f\left(y^{\left(m_{B}\right)}\right) \in D\left(\operatorname{GF}(k)^{m_{B}}, Z_{k}\right)$. Ключами генератора являются начальные состояния $A^{0}, B^{0}$ двух регистров.

Через $A^{i}, B^{i}, i=1,2, \ldots$, обозначим состояния регистров $A, B$ после $i$ шагов регулярного движения.

В такте $i$ вычисляются состояние $A^{i}$ регистра $A$ и значение $u_{i}=u\left(A^{i}\right)$ функции $u$. Затем осуществляется сдвиг регистра $B$ на $u_{i}$ шагов и вычисление состояния $B^{\rho(i)}$ регистра $B$ и знака $f_{\rho(i)}=f\left(B^{\rho(i)}\right)$ результирующей последовательности, где

$$
\rho(i)=\sum_{l=1}^{i} u_{l} .
$$

Выход генератора определяется соотношением

$$
z_{i}=f_{\rho(i)}, i=1, \ldots, t,
$$

где $t$ - число тактов работы генератора. 
Используя вершинные функции графа $Q^{\prime} \in \Delta_{t}^{k}$, заданные соотношениями (3.1), получаем уравнения для знаков результирующей последовательности:

$$
z_{i}=\sum_{j=i \cdot d_{1}}^{i \cdot d_{h}} f_{j} \cdot q_{i, j}^{\prime}\left(u^{(i)}\right), i=1,2, \ldots, t
$$

Если известны $t$ знаков исходной и результирующей последовательностей, то соотношения (4.2) образуют систему ортогонально разложимых квазисимметрических уравнений $S^{\prime} \in S\left(Q^{\prime}, k, t, t\right)$ относительно $t$ неизвестных знаков управляющей последовательности. Все возможные варианты последовательности $t$ знаков управления могут быть перечислены по графу решений системы (4.2).

Вычислительная и ёмкостная сложности построения графа решений системы (4.2) оцениваются согласно утверждению 2) теоремы 3.2 величинами порядка $O\left(t^{3}\right)$ условных операций. При этом наличие искажений в результирующей последовательности не увеличивает трудоёмкость метода, хотя влияет на его надёжность.

4.3. Альтернативный генератор. Альтернативный генератор содержит управляющий регистр $A$ и $k$ регистров $B_{r}, r=0, \ldots, k-1$, исходных последовательностей. Состояниями регистров $A, B_{r}$ являются векторы над полем $\mathrm{GF}(k)$ размерностей $m_{A}, m_{r}$ соответственно. На состояниях регистра $A$ задана аффинная функция $u\left(x^{\left(m_{A}\right)}\right) \in D\left(\operatorname{GF}(k)^{m_{A}}, Z_{k}\right)$, принимающая значения $0,1, \ldots$, $k-1$. На состояниях регистра $B_{r}$ задана функция $f^{(r)}\left(y^{\left(m_{r}\right)}\right) \in$ $\in D\left(\mathrm{GF}(k)^{m_{r}}, Z_{k}\right)$. Регистры $B_{r}$ вырабатывают (при равномерном движении) исходные последовательности $\left\{f_{j}^{(r)}\right\}, r=0, \ldots, k-1, j=1,2 \ldots$.

В такте $i=1,2 \ldots$ вычисляются состояние $A^{i}$ регистра $A$ и значение $u_{i}=$ $=u\left(A^{i}\right)$ функции $u$. Если $u_{i}=r$, то осуществляется сдвиг регистра $B_{r}$ на один шаг и знаком $z_{i}$ результирующей последовательности становится очередной знак последовательности $\left\{f_{j}^{(r)}\right\}, 1 \leq j \leq i$. Другие регистры при этом состояния не меняют.

Ключами генератора являются начальные состояния $A^{0}, B_{r}{ }^{0}$ регистров $A, B_{r}, r=0, \ldots, k-1$.

С учётом сказанного, получаем следующие уравнения образования $t$ знаков результирующей последовательности:

$$
z_{i}=\sum_{r=0}^{k-1} \sum_{j=1}^{h_{i-1}(Q)} f_{s_{r}}^{(r), j} \cdot e_{i-1, j, r}\left(u^{(i)}\right), i=1, \ldots, t .
$$


При заданных отрезках последовательностей $f_{j}^{(r)}, z_{i}$ функции из правых частей системы (4.3) принадлежат модулю $L_{Z}(Q), Q \in \Delta_{t}^{k}$. Соотношения (4.3) образуют систему $S \in S(Q, k, t, t)$ ортогонально разложимых квазисимметрических уравнений. По графу решений системы (4.3) могут быть перечислены все варианты управляющей последовательности.

Вычислительная и ёмкостная сложности построения графа решений системы (4.3) оцениваются по утверждению 1) теоремы 3.2 величинами порядка $O\left(t^{k+1}\right)$ условных операций.

4.4. Определение ключей генераторов. Системы (4.2), (4.3) не являются переопределёнными и, следовательно, могут иметь много решений.

Рассмотрим комбинированный метод определения ключей генераторов, использующий построение графа решений систем (4.2), (4.3) и алгоритм получения аффинных следствий из систем уравнений по графу решений (см. $\$ 5$ статьи [3]).

Аффинным следствием из $H$-системы дискретных уравнений $S$, где $H \in$ $\in \Omega_{n}{ }^{(k)}$, назовём каждое соотношение

$$
\sum_{j=1}^{n} l_{j} \cdot s_{j}=a,
$$

которое выполняется в поле $\mathrm{GF}(k)$ для всех векторов $s^{(n)}=\left(s_{1}, \ldots, s_{n}\right) \in$ $\in \operatorname{sol}(S)$. Множество аффинных следствий из системы $S$ замкнуто относительно сложения и умножения на элементы поля $\mathrm{GF}(k)$ и является, таким образом, векторным пространством над полем $\mathrm{GF}(k)$.

Алгоритм вычисления базиса пространства аффинных следствий из $H$-системы $S$ по её графу решений, предложенный в статье [3], использует параметрическое решение системы $S$.

Напомним, что параметрическим решением совместной $(H, k)$-системы уравнений $S$, где $\left.H \in \Omega_{n}{ }^{(}\right)$, называют каждую систему дискретных функций

$$
\rho_{i}\left(v_{1}, \ldots, v_{m}\right) \in D\left(Z_{k}^{n} ; Z_{k}\right), i=1, \ldots, n,
$$

удовлетворяющую следующим условиям.

$1^{0}$. Если $\left(x_{1}{ }^{(0)}, \ldots, x_{n}{ }^{(0)}\right)$ - решение системы уравнений $S$, то найдутся такие значения $v_{1}{ }^{(0)}, \ldots, v_{m}{ }^{(0)}$, что

$$
x_{i}^{(0)}=\rho_{i}\left(v_{1}{ }^{(0)}, \ldots, v_{m}{ }^{(0)}\right), i=1, \ldots, n .
$$

$2^{0}$. Если для произвольных значений $v_{j} \in Z_{k}, j=1, \ldots, m$ положить

$$
x_{i}=\rho_{i}\left(v_{1}, \ldots, v_{m}\right) \text {, }
$$

то $\left(x_{1}, \ldots, x_{n}\right)$ - решение системы уравнений $S$. 
Сложность предложенного в статье [3] алгоритма построения параметрического решения $H$-системы $S$, зависящей от $n$ неизвестных, по её графу решений, оценивается при $n \rightarrow \infty$ величиной $O(V(G(S)))$ условных операций.

Сложность предложенного там же алгоритма вычисления базиса пространства аффинных следствий из $H$-системы $S$ по её параметрическому решению оценивается при $n \rightarrow \infty$ величиной $O\left(n^{2}\right)$ условных операций.

На первом этапе комбинированного метода определения ключей генераторов составляется система квазисимметрических уравнений и строится граф её решений. На втором этапе по графу решений строится параметрическое решение системы. Это позволяет применить алгоритмы из статьи [3] и получить базис пространства аффинных следствий из системы уравнений.

Далее к базисным аффинным следствиям добавляются уравнения, выражающие рекуррентные зависимости в управляющей последовательности, и находятся решения полученной системы линейных уравнений над полем $\mathrm{GF}(k)$.

Возможные варианты управления даёт пересечение множеств решений линейной системы и исходной системы $S$.

Сложность определения ключей $(1, \ldots, k)$-генератора путём перебора начального заполнения генерирующего регистра с применением в каждом варианте опробования комбинированного метода можно оценить при $t=O\left(m_{A}\right)$ величиной порядка $O\left(k^{m_{B}} \cdot m_{A}^{3}\right)$ условных операций. Для сравнения отметим, что сложность предложенной в [10] алгебраической атаки на аналогичный $(1,2)$-генератор над полем $\mathrm{GF}(2)$ оценивается величиной порядка $O\left(2^{m_{A}} \cdot m_{B}^{3}\right)$ условных операций.

Сложность определения ключей альтернативного генератора путём перебора начальных заполнений генерирующих регистров с применением в каждом варианте опробования комбинированного метода можно оценить при $t=O\left(m_{A}\right)$ величиной порядка $O\left(k^{\sum_{r=1}^{k} m_{r}} \cdot m_{A}^{k+1}\right)$ условных операций. Отметим, что сложность предложенной в [10] алгебраической атаки на аналогичный альтернативный генератор над полем GF(2) оценивается величиной порядка $O\left(2^{m_{A}} \cdot\left(m_{1}^{3}+m_{2}^{3}\right)\right)$ условных операций.

Как видно из приведённых оценок, в случае $k=2$ и при наличии неискажённой выходной последовательности тот или другой вариант атаки может быть более эффективным.

\section{Список литературы}

1. Смирнов В. Г. Методы решения систем дискретных уравнений, основанные на построении графа решений. - В сб.: Труды по дискретной математике. Т. 9. - М.: Гелиос-АРВ, 2006, с. 308-339. 
2. Смирнов В. Г. Эффективные алгоритмы построения графа общего решения для системы булевых уравнений. - В сб.: Труды по дискретной математике. Т. 5. - М.: ФИЗМАТЛИТ, 2002, с. 227-234.

3. Смирнов В. Г. Методы построения графов решений для систем $k$-значных уравнений. - В сб.: Труды по дискретной математике. Т. 8. - М.: ФИЗМАТЛИТ, 2004, с. 253-280.

4. Кормен Т., Лейзерсон Ч., Ривест Р. Алгоритмы: построение и анализ. - М.: МЦНМО, 2001.

5. Балакин Г. В. Введение в теорию случайных систем уравнений, - В сб.: Труды по дискретной математике. Т. 1. - М.: ТВП, 1997, с. 1-18.

6. Биркгоф Г. Теория структур. - М.: ИЛ, 1951.

7. Сачков B. Н. Введение в комбинаторные методы дискретной математики. - М.: Наука, 1982.

8. Поточные шифры. Результаты зарубежной открытой криптологии. M., 1997.

9. Шнайер Б. Прикладная криптография. Протоколы, алгоритмы, исходные тексты на языке Си. - М.: Триумф, 2002.

10. Al-Hinai S., Batten L., Colbert B. Algebraic attacks on clock-controlled stream ciphers. — Lect. Notes Comput. Sci., 2006, v. 4058, p. 1-16. 

